\title{
HIGH-RESOLUTION 4.7 MICRON KECK/NIRSPEC SPECTRA OF PROTOSTARS. I. ICES AND INFALLING GAS IN THE DISK OF L1489 IRS $^{1}$
}

\author{
A. C. A. Boogert, ${ }^{2}$ M. R. Hogerheijde, ${ }^{3,4}$ and G. A. Blake ${ }^{5}$ \\ Received 2001 October 5; accepted 2001 December 4
}

\begin{abstract}
We explore the infrared $M$-band $(4.7 \mu \mathrm{m})$ spectrum of the Class I protostar L1489 IRS in the Taurus molecular cloud. This is the highest-resolution wide-coverage spectrum at this wavelength of a low-mass protostar observed to date $\left(R=25,000 ; \Delta v=12 \mathrm{~km} \mathrm{~s}^{-1}\right)$. A large number of narrow absorption lines of gasphase ${ }^{12} \mathrm{CO},{ }^{13} \mathrm{CO}$, and $\mathrm{C}^{18} \mathrm{O}$ are detected, as well as a prominent band of solid ${ }^{12} \mathrm{CO}$. The gas-phase ${ }^{12} \mathrm{CO}$ lines have redshifted absorption wings (up to $100 \mathrm{~km} \mathrm{~s}^{-1}$ ), which likely originate from warm disk material falling toward the central object. Both the isotopes and the extent of the ${ }^{12} \mathrm{CO}$ line wings are successfully fitted with a contracting-disk model of this evolutionary transitional object. This shows that the inward motions seen in millimeter-wave emission lines continue to within $\sim 0.1 \mathrm{AU}$ of the star. The amount of high-velocity infalling gas is, however, overestimated by this model, suggesting that only part of the disk is infalling, e.g., a hot surface layer or hot gas in magnetic field tubes. The colder parts of the disk are traced by the prominent $\mathrm{CO}$-ice band. The band profile results from $\mathrm{CO}$ in "polar" ices $\left(\mathrm{CO}\right.$ mixed with $\left.\mathrm{H}_{2} \mathrm{O}\right)$ and $\mathrm{CO}$ in "apolar" ices. At high spectral resolution, the "apolar" component is, for the first time, resolved into two distinct components, likely due to pure $\mathrm{CO}$ and $\mathrm{CO}$ mixed with $\mathrm{CO}_{2}, \mathrm{O}_{2}$, and/or $\mathrm{N}_{2}$. The ices have probably undergone thermal processing in the upper disk layer traced by our pencil absorption beam: much of the volatile "apolar" ices has evaporated, the depletion factor of $\mathrm{CO}$ onto grains is remarkably low $(\sim 7 \%)$, and the $\mathrm{CO}_{2}$ traced in the CO-band profile was possibly formed energetically.This study shows that high spectral resolution $4.7 \mu \mathrm{m}$ observations provide important and unique information on the dynamics and structure of protostellar disks and on the origin and evolution of ices in these disks.
\end{abstract}

Subject headings: dust, extinction — infrared: ISM — ISM: molecules — planetary systems: protoplanetary disks — stars: formation stars: individual (L1489 IRS)

\section{INTRODUCTION}

In the process of low-mass star formation, a mixture of gas, dust, and ices accumulates in protostellar envelopes and disks. The fate of this molecular material is diverse. Most of it will fall toward the protostar and dissociate in the inner disk region or stellar photosphere. Some material will be blown away and destroyed by the stellar wind. Some may also survive and be the building material for comets and planets. Major aspects of this complicated process are not well understood and are poorly observationally constrained. For example, do the ices that form comets still resemble ices of the original pristine molecular clouds, or are new ices of different composition being formed in the envelope or disk? The type of ices being formed depends on the composition of the gas that accretes onto grains. Reducing environments produce $\mathrm{H}_{2} \mathrm{O}$-rich ("polar") ices, while in cold, inert environments "apolar" ices rich in $\mathrm{CO}, \mathrm{N}_{2}$, and

\footnotetext{
${ }^{1}$ The data presented herein were obtained at the W. M. Keck Observatory, which is operated as a scientific partnership among the California Institute of Technology, the University of California and the National Aeronautics and Space Administration. The Observatory was made possible by the generous financial support of the W. M. Keck Foundation.

2 Downs Laboratory of Physics 320-47, California Institute of Technology, Pasadena, CA 91125; boogert@submm.caltech.edu.

${ }^{3}$ Radio Astronomy Laboratory, Department of Astronomy, University of California, 601 Campbell Hall 3411, Berkeley, CA 94720.

${ }^{4}$ Current address: Steward Observatory, University of Arizona, 933 North Cherry Avenue, Tucson, AZ 85721.

${ }^{5}$ Division of Geological and Planetary Sciences 150-21, California Institute of Technology, Pasadena, CA 91125.
}

$\mathrm{O}_{2}$ can be formed (Tielens \& Hagen 1982). Depending on the composition, ices evaporate between temperatures of 18 and $90 \mathrm{~K}$. Also, heat can change the solid-state structure of ices by, for example, crystallization. Energetic particles (e.g., cosmic rays) and ultraviolet (UV) radiation are able to initiate reactions in ices and form new species. Dynamics and shocks within disks may be able to destroy ices as well.

Clearly, to determine the relative importance of these ice formation and destruction processes, knowledge of the physical conditions and structure of envelopes and disks is crucial. Much theoretical and observational work on this topic has been done over the last $\sim 10 \mathrm{yr}$. Molecular gas was detected in a suite of protostellar disks by millimeter-wave observations sensitive to emission over radii of several hundred AU (Dutrey, Guilloteau, \& Guélin 1997; Thi et al. 2001). Gas-phase abundances were found to be reduced by factors of 5 to several hundred, depending on the source and the sublimation temperature of the molecules. Models of disk midplanes indeed show high depletions because of the formation of icy mantles on grains (Aikawa et al. 1997; Willacy et al. 1998). The predicted depletions were in fact higher than observed, and thus desorption mechanisms are needed to explain the millimeter-wave observations (Goldsmith, Langer, \& Velusamy 1999). It was realized that the outer parts of disks are heated more efficiently when they are flared (Kenyon \& Hartmann 1987). Thus, by the influence of the stellar radiation a layer with "superheated " dust is formed in which molecules have been dissociated (Chiang $\&$ Goldreich 1997). The layer below that is warm enough to evaporate the ices, but not to dissociate the released molecules. The importance of this warm layer, and the abun- 
dance of gas-phase molecules, depend strongly on how effective ice desorption mechanisms are (Willacy et al. 1998). Recent studies indicate that desorption by UV and $\mathrm{X}$-ray photons may be strong enough to explain observations of molecular gas by millimeter-wave telescopes (Willacy \& Langer 2000; Najita, Bergin, \& Ullom 2001). This idea is confirmed by multitransition molecular-line observations, which indicate temperatures $(>20-40 \mathrm{~K})$ and densities that typically occur in this warm layer (van Zadelhoff et al. 2001). In a third layer, the disk midplane, cold, dense conditions prevail, resulting in extreme depletions of gas-phase molecules many orders of magnitude larger than in quiescent dense molecular clouds. Indeed, recent absorption-line observations failed to detect gas-phase $\mathrm{CO}$ in the edge-on disk around the protostar Elias 18, thus indicating an enormous depletion in the midplane (Shuping et al. 2001).

In this paper we report high spectral resolution ( $R=25,000) 4.7 \mu \mathrm{m} M$-band observations of the obscured protostar L1489 IRS (IRAS 04016+2610) in the Taurus molecular cloud. L1489 IRS is a low-luminosity object (3.7 $L_{\odot}$ ), with a spectral energy distribution resembling that of an embedded Class I protostar (Kenyon, Calvet, \& Hartmann 1993). Detailed millimeter-wave line and continuum studies show that L1489 IRS is surrounded by a large (2000 AU radius), rotating, thick, disklike structure (Hogerheijde et al. 1998; Saito et al. 2001), rather than an inside-out collapsing envelope (Hogerheijde \& Sandell 2000). The rotation is sub-Keplerian, and the disk as a whole is contracting. Thus, it was suggested that L1489 IRS represents a shortlived $\left(2 \times 10^{4} \mathrm{yr}\right)$ transitional phase between embedded YSOs that have large envelopes and small (few hundred $\mathrm{AU})$, rotationally supported disks and $\mathrm{T}$ Tauri stars that have no envelopes and fully rotationally supported 500-800 AU disks (Hogerheijde 2001). This circumstellar (or circumbinary: Wood et al. 2001) disk is seen close to edge-on $\left(60^{\circ}-\right.$ $<90^{\circ}$ ) in scattered-light images (Whitney, Kenyon, \& Gómez 1997; Padgett et al. 1999). A CO outflow emanates from the object (Myers et al. 1988), with Herbig-Haro objects lying along it (Gómez, Whitney, \& Kenyon 1997).

Low spectral resolution infrared observations of L1489 IRS show that deep $\mathrm{H}_{2} \mathrm{O}$ (Sato et al. 1990) and $\mathrm{CO}$ (Chiar et al. 1998; Teixeira, Emerson, \& Palumbo 1998) ice bands are present along the line of sight. In this paper we will use the newly available spectrometer NIRSPEC at the Keck II Telescope to obtain high-resolution $M$-band spectra $(\Delta v=12$ $\mathrm{km} \mathrm{s}^{-1}$ at $\left.4.7 \mu \mathrm{m}\right)$ of this source. The large array of NIRSPEC allows both the vibrational band of solid $\mathrm{CO}$ and the surrounding rovibrational transitions of gas-phase $\mathrm{CO}$ to be observed in the same high-resolution spectrum. This offers a new view of this system, on both the origin and evolution of ices and the interrelationship of gas and ices, as well as on the kinematics and structure of the young, contracting, close to edge-on disk. It is a unique view, because infrared absorption-line studies trace all gas and solid-state material at all radii from the star, while present-day millimeter-wave observations are limited by their relatively low spatial resolution $(\geq 100 \mathrm{AU})$. Thus, one of the questions that will be answered in this paper is whether the large-scale inward motions seen in millimeter-wave emission lines continue to smaller radii (1 AU or less) from the star.

Previous studies have already shown that rich astrophysical information can be obtained from high spectral resolution observations in the atmospheric $M$ band. Mainly massive, luminous protostars were observed, however
(Mitchell et al. 1990), and the few observations of low-mass protostars cover a small wavelength range containing only a few gas-phase lines and not the solid CO band (Shuping et al. 2001; Carr, Mathieu, \& Najita 2001). This is Paper I in a series on high-resolution $M$-band spectroscopy of protostars, initiated by the availability of the NIRSPEC spectrometer at Keck II, with which weak, low-mass protostars can be routinely observed at high spectral resolution over a large wavelength range, covering both the solid and gasphase $\mathrm{CO}$ features.

The reduction of the long-slit spectra is discussed in $\S 2$. In $\S 3.1$, we analyze the observed gas lines, which at this resolution even give dynamical information. We use standard curve-of-growth and rotation-diagram techniques to obtain a first idea of gas column and temperatures. In order to analyze the solid CO-band profile in this line of sight, a detailed discussion of available laboratory experiments of solid $\mathrm{CO}$ is given in $\S 3.2$. In $\S 4$.1, we apply the infalling-disk model of Hogerheijde (2001) to explain the observed gas-phase ${ }^{12} \mathrm{CO}$ and ${ }^{13} \mathrm{CO}$ line profiles and constrain the physical conditions and structure of the disk. The possibility of binarity is briefly discussed in $\S 4.2$. The gas-phase analysis is linked to the solid $\mathrm{CO}$ results to determine the origin and thermal history of solid CO in $\S 4.3$. We conclude with suggestions for future work in $\S 5$.

\section{OBSERVATIONS}

The infrared source L1489 IRS was observed with the NIRSPEC spectrometer (McLean et al. 1998) at the Keck II Telescope atop Mauna Kea on the nights of 2001 January $28 / 29$ and 29/30. The sky was constantly clear and dry, and the seeing was reasonable $(\sim 0.5-0 " 7$ at $2.2 \mu \mathrm{m})$. NIRSPEC was used in the echelle mode with the $0.43 \times 24^{\prime \prime}$ slit, providing a resolving power of $R=\lambda / \Delta \lambda=25,000(\sim 12 \mathrm{~km}$ $\left.\mathrm{s}^{-1}\right),{ }^{6}$ with three Nyquist-sampled settings covering the wavelength range $4.615-4.819 \mu \mathrm{m}$ in the atmospheric $M$ transmission band.

The data were reduced in a standard way, using IDL routines. The thermal background emission was removed by differencing the nodding pair. Each nodding position was integrated on for 1 minute, before the telescope was pointed to the other nodding position. The positioning had to be done by hand, because condensations on the Dewar window prevented us from using the image rotator, and the instrument had to be used in the nonstandard "stationary guiding mode." In this mode, the condensations were well removed by subtracting nodding pairs, although the sensitivity of Keck/NIRSPEC was reduced by several magnitudes.

The most critical step in the reduction of these data is correction for atmospheric absorption features and separate telluric and interstellar CO absorption features. For our observations of L1489 IRS this proved to be relatively easy, because at the time of our observations the deep interstellar CO lines were shifted by as much as $43 \mathrm{~km} \mathrm{~s}^{-1}$ with respect to telluric $\mathrm{CO}$ lines. The low air mass of the source, 1.001.05, also made this step easier. The standard stars HR 1380 (A7 V) and HR 1497 (B3 V) were observed at similar air mass, and their spectral shape and hydrogen absorption features were divided out with Kurucz model atmospheres. A

\footnotetext{
${ }^{6}$ This nominal spectral resolution was verified by measuring the Gaussian width of absorption lines in a variety of astrophysical sources.
} 
good telluric correction was achieved, although residuals are seen near deep atmospheric lines. To be safe, we therefore removed the parts of the spectrum that have less than $50 \%$ of the maximum transmission in each setting. This does not affect most of the features in the L1489 IRS spectrum, because the velocity shift of $43 \mathrm{~km} \mathrm{~s}^{-1}$ fully separates them from telluric features. The final signal-to-noise ratio on the unsmoothed spectrum is $\sim 45$, after integration times of 11 , 14 , and 20 minutes on each of the three settings of this $M=4.8 \mathrm{mag}$ (Kenyon et al. 1993) source. The different settings were wavelength-calibrated on the atmospheric $\mathrm{CO}$ emission lines, and subsequently the three settings were combined by applying relative multiplication factors. We have not attempted to flux-calibrate the spectrum, because we are interested in absorption features only.

\section{RESULTS}

The fully reduced echelle spectrum of L1489 IRS shows, in great detail, many deep, narrow absorption lines of gasphase ${ }^{12} \mathrm{CO}$ and ${ }^{13} \mathrm{CO}$ and a few weak lines of $\mathrm{C}^{18} \mathrm{O}$ (Fig. 1). These lines were identified using the line frequencies in the HITRAN catalog (Rothman et al. 1992). The broad absorption feature between 2122 and $2149 \mathrm{~cm}^{-1}$ can be attributed to the stretching vibration mode of ${ }^{12} \mathrm{CO}$ in ices along this line of sight.

In order to analyze these gas and solid-state absorption features, a shallow, second-order polynomial continuum was applied to derive the optical depth spectrum. The solid $\mathrm{CO}$ band was then analyzed using available laboratory experiments ( $\S 3.2$ ). The derivation of physical parameters from the gas-phase lines is highly model-dependent. First, we will derive temperatures and column densities using the standard curve-of-growth and rotation-diagram techniques (§ 3.1). Then we will independently test an astrophysically relevant power-law model in $\S 4$.1. This information is combined in $\S 4.3$ to discuss the origin and thermal history of the solid CO seen in this line of sight.

\subsection{Gas-Phase $\mathrm{CO}$}

The ${ }^{12} \mathrm{CO}$ lines have a complicated profile. Deep lines are present at a velocity of $43 \mathrm{~km} \mathrm{~s}^{-1}$ with respect to earth, which is the systemic velocity of L1489 IRS at the date these observations were made (taking $v_{\mathrm{lsr}}=5 \mathrm{~km} \mathrm{~s}^{-1}$ from millimeter emission lines; see, e.g., Hogerheijde \& Sandell 2000). Each of the ${ }^{12} \mathrm{CO}$ lines is accompanied by a fairly prominent wing at the redshifted side (Fig. 2). As an exploring step in the analysis, we decomposed the main ${ }^{12} \mathrm{CO}$ component and its wing by fitting two Gaussians. They are separated by, on average, $23 \pm 6 \mathrm{~km} \mathrm{~s}^{-1}$, and the main feature and its wing have widths of FWHM $=20 \pm 3$ and $53 \pm 17 \mathrm{~km} \mathrm{~s}^{-1}$, respectively. The main ${ }^{12} \mathrm{CO}$ feature at the systemic velocity is resolved. Excess absorption is visible at the blue and red sides with respect to ${ }^{13} \mathrm{CO}$ (Fig. 2). The ${ }^{13} \mathrm{CO}$ lines have Gaussian shapes, with a width equal to the instrumental resolution $\left(\mathrm{FWHM}=12 \mathrm{~km} \mathrm{~s}^{-1}\right)$. The main absorption feature of the ${ }^{12} \mathrm{CO}$ lines is therefore only in part responsible for the same gas seen in ${ }^{13} \mathrm{CO}$.

We derived equivalent widths for the isotopes and ${ }^{12} \mathrm{CO}$ components (Tables 1 and 2) and applied a standard curveof-growth technique to calculate column densities for each

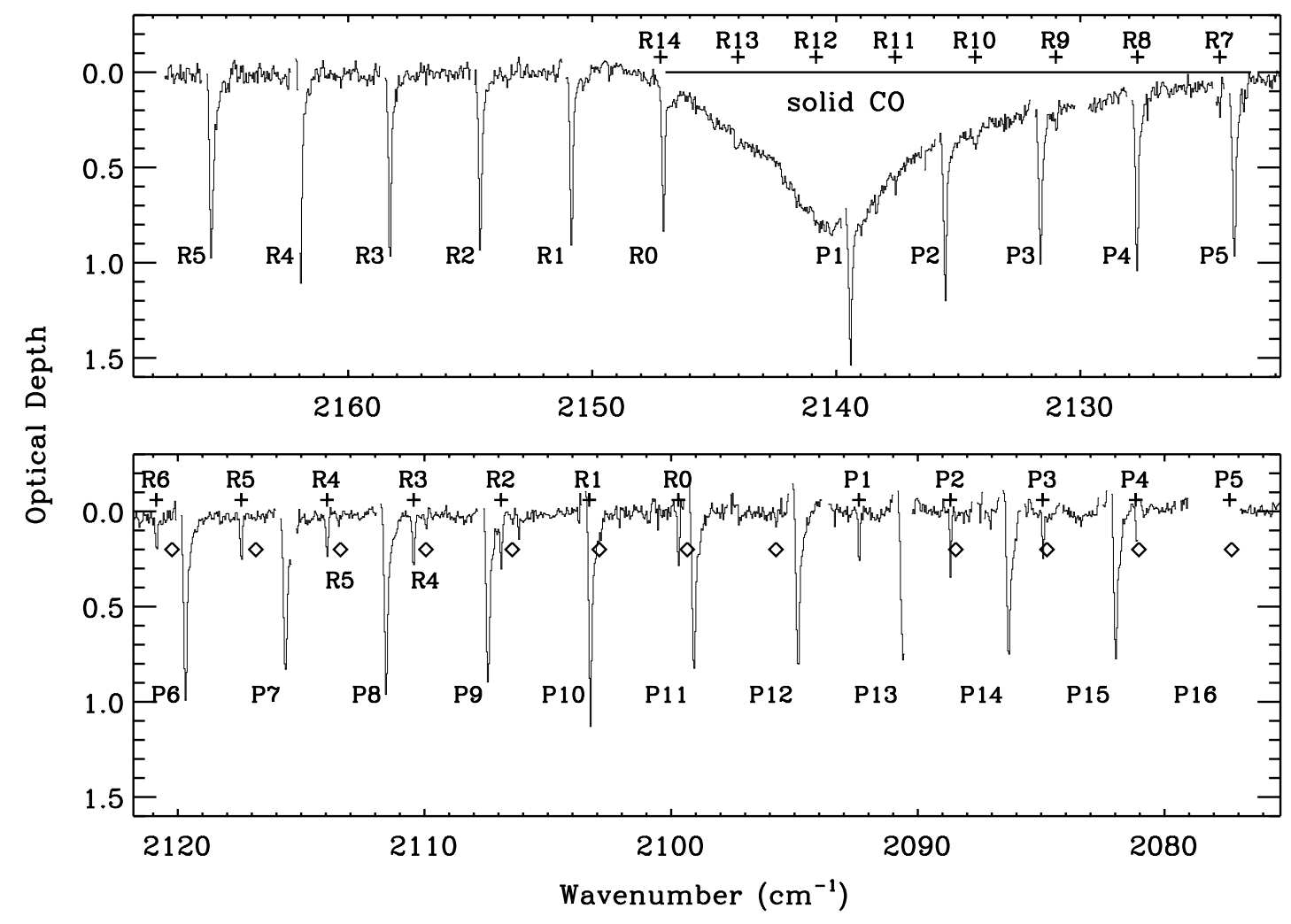

FIG. 1.-Observed, unsmoothed, $R=25,000 \mathrm{M}$-band spectrum of L1489 IRS, corrected for object and earth velocity $\left(43 \mathrm{~km} \mathrm{~s}^{-1}\right)$ on optical depth scale. Gas-phase line frequencies of ${ }^{12} \mathrm{CO}$ (deepest lines) and ${ }^{13} \mathrm{CO}$ (plus signs above spectrum) determined from the HITRAN database (Rothman et al. 1992) are labeled. Diamonds indicate the expected position of $\mathrm{C}^{18} \mathrm{O}$ absorption lines, with the $R(4)$ and $R(5)$ line detections labeled. The prominent broad absorption between 2123 and $2149 \mathrm{~cm}^{-1}$ can be fully ascribed to solid CO. Wavelength regions with poor atmospheric transmission are not plotted. 


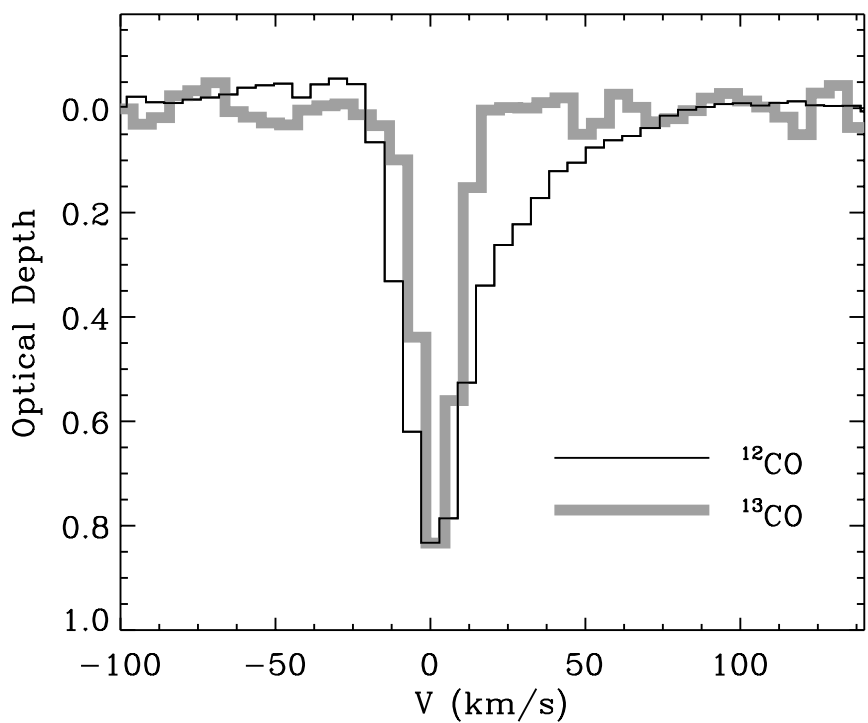

FIG. 2.-Comparison of the ${ }^{12} \mathrm{CO}$ (black line) and ${ }^{13} \mathrm{CO}$ (gray line) spectra of L1489 IRS. Spectra are averaged over the observed ${ }^{12} \mathrm{CO} P(6)-P(15)$ lines and all ${ }^{13} \mathrm{CO}$ lines, in order to increase the signal-to-noise ratio. They are corrected for the source and earth velocity $\left(43 \mathrm{~km} \mathrm{~s}^{-1}\right)$, and the ${ }^{13} \mathrm{CO}$ spectrum was multiplied by 3.64 to facilitate comparison.

$J$-level. The comparison of equal $J$-levels of $\mathrm{C}^{18} \mathrm{O},{ }^{13} \mathrm{CO}$, and the main ${ }^{12} \mathrm{CO}$ component provides a handle on the intrinsic line width $b_{D}\left[=\mathrm{FWHM} / 2(\ln 2)^{1 / 2}\right]$, on the assumption that all material absorbs at the same velocity (however, see $\S 4.1$ ). One also has to assume that the isotope ratios are constant along the line of sight $\left({ }^{12} \mathrm{CO} /{ }^{13} \mathrm{CO}=80\right.$ and ${ }^{12} \mathrm{CO} / \mathrm{C}^{18} \mathrm{O}=560$; Wilson \& Rood 1994). The equivalent widths of $\mathrm{C}^{18} \mathrm{O}$ and ${ }^{13} \mathrm{CO}$ are then simultaneously fitted at $b_{D}>0.8 \mathrm{~km} \mathrm{~s}^{-1}$. Lower $b_{D}$ significantly $(>3 \sigma)$ underesti-

TABLE 1

${ }^{12}$ CO Equivalent Widths

\begin{tabular}{ccc}
\hline \hline Transition & $\begin{array}{c}W_{\nu}, \text { main }^{\mathrm{a}} \\
\left(10^{-3} \mathrm{~cm}^{-1}\right)\end{array}$ & $\begin{array}{c}W_{\nu}, \mathrm{wing}^{\mathrm{a}} \\
\left(10^{-3} \mathrm{~cm}^{-1}\right)\end{array}$ \\
\hline$R(5) \ldots \ldots \ldots \ldots$. & $88(11)$ & $70(22)$ \\
$R(4) \ldots \ldots \ldots \ldots$. & $84(10)$ & $54(17)$ \\
$R(3) \ldots \ldots \ldots \ldots$. & $67(9)$ & $55(18)$ \\
$R(2) \ldots \ldots \ldots \ldots$. & $73(10)$ & $64(20)$ \\
$R(1) \ldots \ldots \ldots \ldots$. & $70(9)$ & $39(12)$ \\
$R(0) \ldots \ldots \ldots \ldots$. & $68(9)$ & $\ldots$ \\
$P(1) \ldots \ldots \ldots \ldots$. & $65(9)$ & $11(4)$ \\
$P(2) \ldots \ldots \ldots \ldots$. & $72(10)$ & $30(10)$ \\
$P(3) \ldots \ldots \ldots \ldots$. & $80(11)$ & $21(7)$ \\
$P(4) \ldots \ldots \ldots \ldots$. & $69(9)$ & $82(26)$ \\
$P(5) \ldots \ldots \ldots \ldots$. & $84(11)$ & $73(23)$ \\
$P(6) \ldots \ldots \ldots \ldots$. & $85(10)$ & $65(20)$ \\
$P(7) \ldots \ldots \ldots \ldots$. & $85(11)$ & $90(28)$ \\
$P(8) \ldots \ldots \ldots \ldots$. & $85(11)$ & $71(22)$ \\
$P(9) \ldots \ldots \ldots \ldots$. & $85(11)$ & $69(21)$ \\
$P(10) \ldots \ldots \ldots \ldots$. & $86(10)$ & $84(26)$ \\
$P(11) \ldots \ldots \ldots \ldots$. & $79(10)$ & $61(18)$ \\
$P(12) \ldots \ldots \ldots \ldots$. & $79(11)$ & $56(17)$ \\
$P(13) \ldots \ldots \ldots \ldots$. & $88(12)$ & $43(13)$ \\
$P(14) \ldots \ldots \ldots \ldots$. & $69(9)$ & $64(19)$ \\
$P(15) \ldots \ldots \ldots \ldots$. & $72(10)$ & $53(16)$ \\
$P(16) \ldots \ldots \ldots$. & $\ldots .$. & $\ldots$ \\
\hline
\end{tabular}

a Values in parentheses are $1 \sigma$ errors.
TABLE 2

${ }^{13} \mathrm{CO}$ and $\mathrm{C}^{18} \mathrm{O}$ Equivalent Widths

\begin{tabular}{|c|c|}
\hline Transition & $\begin{array}{c}W_{\nu}^{\mathrm{a}} \\
\left(10^{-3} \mathrm{~cm}^{-1}\right)\end{array}$ \\
\hline \multicolumn{2}{|l|}{${ }^{13} \mathrm{CO}$} \\
\hline ............... & $<3$ \\
\hline$R(16) \ldots \ldots \ldots \ldots \ldots \ldots \ldots$ & $<5$ \\
\hline 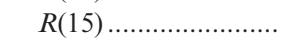 & $5(2)$ \\
\hline$R(14) \ldots \ldots \ldots \ldots \ldots \ldots \ldots$ & $\ldots$ \\
\hline$R(13) \ldots \ldots \ldots \ldots \ldots \ldots \ldots$ & $5(2)$ \\
\hline$R(12) \ldots \ldots \ldots \ldots \ldots \ldots \ldots$ & $<4$ \\
\hline$R(11) \ldots \ldots \ldots \ldots \ldots \ldots$ & $6(2)$ \\
\hline$R(10) \ldots \ldots \ldots \ldots \ldots \ldots$ & $6(2)$ \\
\hline$R(9) \ldots \ldots \ldots \ldots \ldots \ldots \ldots \ldots$ & $7(2)$ \\
\hline$R(8) \ldots \ldots \ldots \ldots \ldots \ldots$ & $\ldots$ \\
\hline$R(7) \ldots \ldots \ldots \ldots \ldots \ldots$ & $12(3)$ \\
\hline$R(6) \ldots \ldots \ldots \ldots \ldots \ldots \ldots$ & $13(2)$ \\
\hline$R(5) \ldots \ldots \ldots \ldots \ldots \ldots \ldots \ldots$ & $19(2)$ \\
\hline$R(4) \ldots \ldots \ldots \ldots \ldots \ldots \ldots \ldots \ldots$ & $17(2)$ \\
\hline$R(3) \ldots \ldots \ldots \ldots \ldots \ldots \ldots \ldots$ & $21(2)$ \\
\hline$R(2) \ldots \ldots \ldots \ldots \ldots \ldots \ldots \ldots \ldots \ldots \ldots \ldots$ & $18(2)$ \\
\hline$R(1)$ & \\
\hline$R(0)$ & $24(2)$ \\
\hline$P(1)$ & $22(2)$ \\
\hline$P(2) \ldots \ldots \ldots \ldots \ldots \ldots \ldots \ldots \ldots$ & $25(2)$ \\
\hline$P(3) \ldots \ldots \ldots \ldots \ldots \ldots \ldots$ & $18(2)$ \\
\hline$P(4) \ldots \ldots \ldots \ldots \ldots \ldots$ & $15(2)$ \\
\hline \multicolumn{2}{|l|}{$\mathrm{C}^{18} \mathrm{O}$} \\
\hline$R(6) \ldots \ldots \ldots \ldots \ldots \ldots \ldots$ & $<3$ \\
\hline$R(5) \ldots \ldots \ldots \ldots \ldots \ldots \ldots \ldots \ldots \ldots \ldots \ldots \ldots \ldots$ & $6(2)$ \\
\hline$R(4) \ldots \ldots \ldots \ldots \ldots \ldots \ldots \ldots$ & $6(2)$ \\
\hline$R(3) \ldots \ldots \ldots \ldots \ldots \ldots \ldots \ldots$ & $<5$ \\
\hline$R(2) \ldots \ldots \ldots \ldots \ldots \ldots \ldots \ldots \ldots$ & $\ldots$ \\
\hline$R(1) \ldots \ldots \ldots \ldots \ldots \ldots$ & $\ldots$ \\
\hline$R(0) \ldots \ldots \ldots \ldots \ldots \ldots \ldots \ldots \ldots \ldots \ldots \ldots \ldots$ & $<6$ \\
\hline$P(1) \ldots \ldots \ldots \ldots \ldots \ldots \ldots$ & $<3$ \\
\hline$P(2) \ldots \ldots \ldots \ldots \ldots \ldots$ & $<3$ \\
\hline
\end{tabular}

a Values in parentheses are $1 \sigma$ errors.

mates the ${ }^{13} \mathrm{CO}$ lines with respect to $\mathrm{C}^{18} \mathrm{O}$. As mentioned above, the main ${ }^{12} \mathrm{CO}$ component is clearly contaminated by gas not seen in ${ }^{13} \mathrm{CO}$, on both the blueshifted and redshifted sides. As a first-order correction, we lowered the ${ }^{12} \mathrm{CO}$ equivalent widths in Table 1 by $40 \%$, which corresponds to unresolved lines at the observed peak optical depth. Then we find that all isotopes are best fitted simultaneously at $b_{D}=1.4 \pm 0.1 \mathrm{~km} \mathrm{~s}^{-1}$. This would be an upper limit if the correction for contamination of the ${ }^{12} \mathrm{CO}$ lines were too small. Hence, this curve-of-growth analysis of the $\mathrm{CO}$ isotopes shows that, on the assumption that all gas absorbs at the same velocity, $b_{D}$ is limited to $0.8 \mathrm{~km}$ $\mathrm{s}^{-1}<b_{D}<1.5 \mathrm{~km} \mathrm{~s}^{-1}$.

With the column densities per $J$-level at hand, a rotation diagram was constructed for the ${ }^{13} \mathrm{CO}$ lines (Fig. 3), to derive the total column density and temperature at a number of allowed $b_{D}$-values (Table 3). Clearly, the rotation diagram shows a double temperature structure, much resembling that of high-mass objects (Mitchell et al. 1990): cold $(T \sim 15 \mathrm{~K})$ and warm gas $(T \sim 250 \mathrm{~K})$ are present along the same line of sight. The column density of the cold component toward L1489 IRS is a particularly strong function of $b_{D}$, increasing by an order of magnitude from 0.7 to $1.3 \mathrm{~km} \mathrm{~s}^{-1}$. An independent $\mathrm{CO}$ column of $1.4 \times 10^{19} \mathrm{~cm}^{-2}$ can be estimated from $A_{V}=29$ (Myers et al. 1987), assum- 


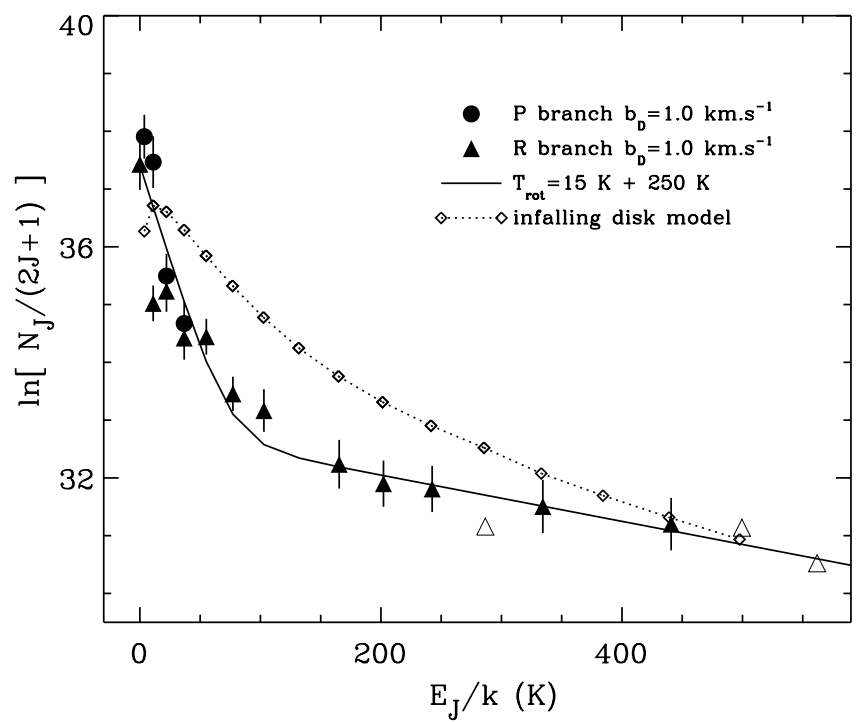

FIg. 3.-Rotation diagram of ${ }^{13} \mathrm{CO}$. Column densities on the vertical axis are calculated from the curve of growth, with $b_{D}=1.0 \mathrm{~km} \mathrm{~s}^{-1}$. Circles: $P$ branch; filled triangles: $R$ branch; open triangles: $R$-branch upper limits. The solid line corresponds to temperatures $T_{\text {rot }}=15 \mathrm{~K}$ and $250 \mathrm{~K}$ (Table 3 ) in the Boltzmann equation. Diamonds along the dotted line represent the rotation diagram for our disk model, which is different from the curve-ofgrowth analysis because of a lower assumed $b_{D}\left(0.1 \mathrm{~km} \mathrm{~s}^{-1}\right)$ and the presence of a velocity gradient $(\S 4.1)$, although both models fit the observed ${ }^{13} \mathrm{CO}$ lines well.

ing that the dust and gas are cospatial. This would suggest that $b_{D}$ is in the $1.0-1.3 \mathrm{~km} \mathrm{~s}^{-1}$ range.

For the highly redshifted ${ }^{12} \mathrm{CO}$ wing a rotation diagram is constructed as well. Here we assume that the absorption is optically thin, because the wings are absent in ${ }^{13} \mathrm{CO}$, as evidenced by the high signal-to-noise ratio average line profile (Fig. 2). The rotational temperature of this highly redshifted gas is similar to that of the warm ${ }^{13} \mathrm{CO}$ component $\left(250_{-100}^{+500}\right.$ $\mathrm{K})$; however, the column is an order of magnitude lower (Table 3). Finally, assuming that the absorption in the wings of the resolved ${ }^{12} \mathrm{CO}$ main component is optically thin, the column of this blue- and redshifted low-velocity gas (within $10 \mathrm{~km} \mathrm{~s}^{-1}$ of the systemic velocity) is about $25 \%$ of the column of the high-velocity redshifted gas at a similar, although poorly determined, temperature.

Summarizing, this basic analysis indicates that, from ${ }^{13} \mathrm{CO}$, both a large column of cold $15 \mathrm{~K}$ gas and a significant amount of warm gas $(T \sim 250 \mathrm{~K})$ are present within $\sim 3 \mathrm{~km}$ $\mathrm{s}^{-1}$ of the systemic velocity (Table 3 ). The ${ }^{12} \mathrm{CO}$ lines show that warm gas at $T \sim 250 \mathrm{~K}$ is also present at highly redshifted velocities $\left(20-100 \mathrm{~km} \mathrm{~s}^{-1}\right)$, but at a factor of 10 lower column. A small amount of warm gas is present at low redand blueshifted velocities as well (within $10 \mathrm{~km} \mathrm{~s}^{-1}$ ). As further described in $\S 4.1$, the gas components at the redshifted and systemic velocities can be fitted within the same physical model of a contracting disk, but the origin of the warm gas at low blueshifted velocities is more difficult to explain.

\subsection{Solid CO}

The broad absorption feature between 2122 and 2149 $\mathrm{cm}^{-1}$ (Fig. 1) can be entirely attributed to the stretching vibration mode of ${ }^{12} \mathrm{CO}$ in circumstellar ices $(\S 4.3)$. The high spectral resolution allows, for the first time, the unambiguous separation of the gas-phase $\mathrm{CO}$ lines from the solid-state absorption and the study of the solid CO-band profile in great detail. In accordance with previous, low-resolution studies (Chiar et al. 1998; Teixeira et al. 1998), a distinct narrow feature is seen at $2140 \mathrm{~cm}^{-1}$ and a significantly broader component at longer wavelengths. Our data, however, indicate the presence of a new, third component on the blue side, separate from the narrow $2140 \mathrm{~cm}^{-1}$ feature, most notable by a change of the blue slope at $2142 \mathrm{~cm}^{-1}$ (Fig. 1).

In order to explain the shape of this $\mathrm{CO}$ absorption profile, we have taken laboratory experiments with solid $\mathrm{CO}$ from the literature (Sandford et al. 1988; Schmitt, Greenberg, \& Grim 1989; Palumbo \& Strazzulla 1993; Trotta 1996; Ehrenfreund et al. 1997; Elsila, Allamandola, \& Sandford 1997; Baratta \& Palumbo 1998; Teixeira et al. 1998). We determined the peak position and width of the laboratory profiles as a function of ice composition, temperature, and cosmic-ray bombardment intensity. Additionally, for CO-rich ices we used optical constants to calculate the absorption profile as a function of particle shape in the small-particle Rayleigh limit (for details, see Ehrenfreund et al. 1997). The absorption profile of CO-poor ices (concentration $<30 \%$ ) is not affected by these particle-shape effects. Changing these various parameters gives a wide variety of peak positions (2135-2144 $\left.\mathrm{cm}^{-1}\right)$ and widths $\left(\mathrm{FWHM}=1.5-14 \mathrm{~cm}^{-1}\right)$ in the laboratory. We limit ourselves here by identifying general trends, as summarized in Figure 4. These trends are then related to the Gaussian peak position and width of the three aforementioned components observed toward L1489 IRS (also given in Fig. 4).

The absorption band of a thin film of pure, solid $\mathrm{CO}$ at $T=10 \mathrm{~K}$ peaks at $\sim 2139 \mathrm{~cm}^{-1}$ and is very narrow $(\sim 2$ $\mathrm{cm}^{-1}$ ). This clearly does not fit any of the components

TABLE 3

Physical Parameters From Curve of Growth and Rotation Diagram

\begin{tabular}{|c|c|c|c|c|}
\hline $\begin{array}{l}T_{\text {rot }} \\
(\mathrm{K})\end{array}$ & $\begin{array}{c}b_{D} \\
\left(\mathrm{~km} \mathrm{~s}^{-1}\right)\end{array}$ & $\begin{array}{c}N\left({ }^{12} \mathrm{CO}\right)^{\mathrm{a}} \\
\left(10^{18} \mathrm{~cm}^{-2}\right)\end{array}$ & $\begin{array}{c}v_{\text {lsr }} \\
\left(\mathrm{km} \mathrm{s}^{-1}\right)\end{array}$ & Origin \\
\hline $19_{-4}^{+5} \ldots \ldots \ldots \ldots \ldots$ & 1.6 & 6 & $5 \pm 3$ & Disk plus foreground; from ${ }^{13} \mathrm{CO}$ \\
\hline $15_{-3}^{+8}$ & 1.3 & 10 & $5 \pm 3$ & As above, but with alternative $b_{D}$ \\
\hline $13_{-3}^{+8} \ldots \ldots \ldots \ldots \ldots$ & 1.0 & 19 & $5 \pm 3$ & As above, but with alternative $b_{D}$ \\
\hline $11_{-3}^{+8} \ldots \ldots \ldots \ldots \ldots \ldots \ldots$ & 0.7 & 96 & $5 \pm 3$ & As above, but with alternative $b_{D}$ \\
\hline 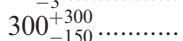 & 1.3 & 2.4 & $5 \pm 3$ & Disk; from ${ }^{13} \mathrm{CO}$ \\
\hline $250_{-100}^{+300} \cdots \cdots \cdots \cdots$ & $1.0 / 0.7$ & 2.8 & $5 \pm 3$ & As above, but with alternative $b_{D}$ \\
\hline $250_{-100}^{+100} \ldots \ldots \ldots \ldots$ & $<32$ & $0.20 \pm 0.03^{\mathrm{b}}$ & $28 \pm 6$ & ${ }^{12} \mathrm{CO}$ red wing \\
\hline $500 \pm 250 \ldots \ldots$ & $<12$ & $\sim 0.06^{\mathrm{b}}$ & $-5,15$ & Disk? From blue and red wings-resolved ${ }^{12} \mathrm{CO}$ main component \\
\hline
\end{tabular}

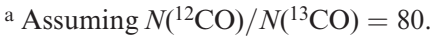

b Assuming optically thin absorption. 


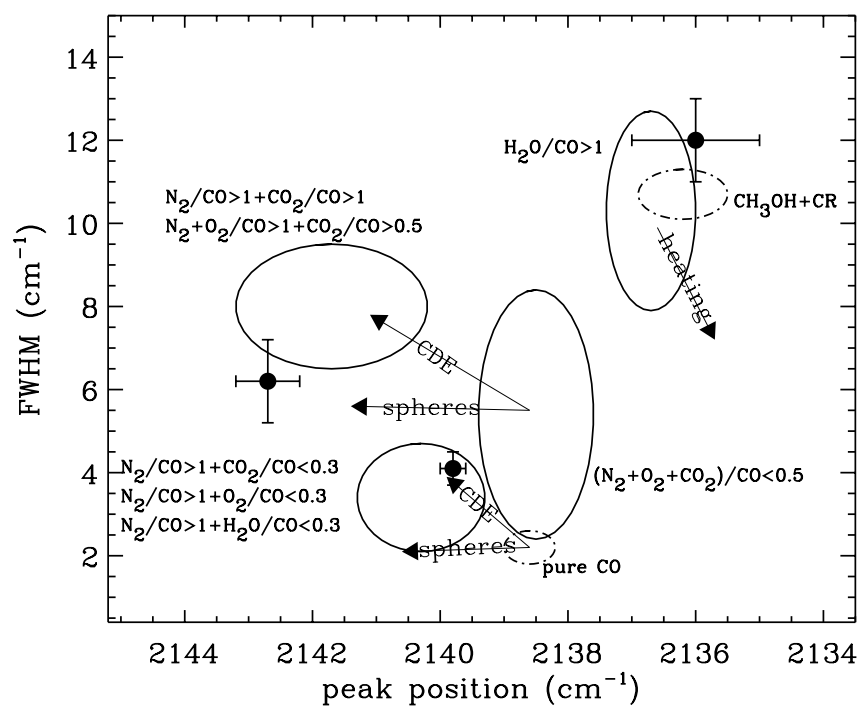

FIG. 4.-Effect of several astrophysically relevant parameters on the peak position and width of the solid ${ }^{12} \mathrm{CO}$ stretching mode, as determined from laboratory experiments. Ellipses indicate ices of various composition. For CO-rich ices, the effect of grain shapes is indicated by arrows for spheres and ellipsoids (CDE). For polar ices, the effect (magnitude and direction) of heating is shown by the arrow. $\mathrm{CH}_{3} \mathrm{OH}+\mathrm{CR}$ means a $\mathrm{CH}_{3} \mathrm{OH}$ or a $\mathrm{CH}_{3} \mathrm{OH}: \mathrm{H}_{2} \mathrm{O}$ ice irradiated by energetic particles simulating cosmic rays. Circles with error bars represent the observed peak position and width of the absorption components detected toward L1489 IRS.

observed toward L1489 IRS. However, for ellipsoidally shaped grains (more precisely, a distribution of ellipsoidal shapes, "CDE"; Bohren \& Huffman 1983), this sensitive, strong band shifts to shorter wavelengths and becomes broader. Now it exactly fits the $2140 \mathrm{~cm}^{-1}$ component observed toward L1489 IRS, both in peak position and width (Fig. 4). In view of a recent controversy on optical constants (Ehrenfreund et al. 1997), it is worth noting that good fits are obtained with optical constants from the works of Ehrenfreund et al. (1997) and Baratta \& Palumbo (1998). The optical constants of Trotta (1996) do not induce strong particle-shape effects, and therefore pure $\mathrm{CO}$ does not provide a good fit to L1489 IRS for any particle shape.

Broadening of the laboratory profile, in order to fit the $2140 \mathrm{~cm}^{-1}$ feature in L1489 IRS, is also achieved by adding a small amount of $\mathrm{CO}_{2}, \mathrm{O}_{2}$, or $\mathrm{H}_{2} \mathrm{O}$ molecules. To avoid too large a broadening, and to minimize the aforementioned particle-shape effects, this mixture must be diluted in $\mathrm{N}_{2}$. This astrophysically relevant molecule does not broaden the feature and gives a small blueshift (Ehrenfreund et al. 1997; Elsila et al. 1997), required to fit the $2140 \mathrm{~cm}^{-1}$ feature in L1489 IRS. Thus, both this mixture and ellipsoidally shaped pure CO-ice grains provide good fits to the central 2140 $\mathrm{cm}^{-1}$ feature.

Now the width is significantly increased and the peak shifted to longer wavelengths by diluting $\mathrm{CO}$ in a mixture of molecules with large dipole moments, such as $\mathrm{H}_{2} \mathrm{O}$ or $\mathrm{CH}_{3} \mathrm{OH}$ (Sandford et al. 1988; Tielens et al. 1991). This particular behavior is needed to fit the broad long-wavelength wing seen toward L1489 IRS. Solid $\mathrm{CH}_{3} \mathrm{OH}$ has an abundance less than a few percent of solid $\mathrm{H}_{2} \mathrm{O}$ toward low-mass objects (Chiar, Adamson, \& Whittet 1996). $\mathrm{H}_{2} \mathrm{O}$ seems the best dilutant, because of its large interstellar abundance. The use of $\mathrm{H}_{2} \mathrm{O}$ : $\mathrm{CO}$ mixtures requires interesting additional constraints. Although a low-temperature, unpro- cessed, $\mathrm{H}_{2} \mathrm{O}$-rich ice does provide a good fit to the red wing, it can be excluded based on the presence of a prominent second absorption at $\sim 2150 \mathrm{~cm}^{-1}$ in the laboratory, which is clearly absent toward L1489 IRS. This second peak is caused by $\mathrm{CO}$ molecules located in pockets in an amorphous ice. These CO molecules are weakly bound, and the $\sim 2150 \mathrm{~cm}^{-1}$ peak disappears rapidly at higher $T$, or as a result of cosmic-ray hits (Sandford et al. 1988). Thus, the $\mathrm{H}_{2} \mathrm{O}$ ice responsible for the long-wavelength wing toward L1489 IRS must be thermally $(T>50 \mathrm{~K})$ or energetically processed.

The blue wing seen at $\sim 2143 \mathrm{~cm}^{-1}$ toward L1489 IRS can only be explained by an apolar ice. Adding a significant amount of $\mathrm{CO}_{2}$ to a $\mathrm{CO}$ ice $\left(\mathrm{CO}_{2} / \mathrm{CO}>1\right)$ results in the blueshift and broadening required to fit the observed wing. Somewhat less $\mathrm{CO}_{2}$ is needed $\left(\mathrm{CO}_{2} / \mathrm{CO} \sim 0.5\right)$ when a large amount of $\mathrm{O}_{2}$ is present. $\mathrm{N}_{2}$ may be added as well, but it is not essential except as a dilutant to reduce the effects of particle shape. A good fit is obtained by the mixture $\mathrm{N}_{2}: \mathrm{O}_{2}: \mathrm{CO}_{2}: \mathrm{CO}=1: 5: 0.5: 1$, as proposed in Elsila et al. (1997). If $\mathrm{CO}_{2} / \mathrm{CO}<0.5$, the band peaks at too high a wavelength. In CO-rich ices this problem can, however, be overcome by particle-shape effects (Fig. 4). In view of this effect, it is not possible to constrain the relative molecular abundances of this interstellar component in more detail, but it is clear that an apolar $\mathrm{CO}_{2}$ or $\mathrm{O}_{2}$ ice is needed, different from the distinct $2140 \mathrm{~cm}^{-1}$ feature.

A three-component fit to the entire CO-ice band of L1489 IRS is shown in Figure 5. Although this is not a unique fit, it does obey the global trends that we identified in the laboratory experiments.

Finally, the solid CO column density is derived by dividing the integrated optical depth by the band strength $A$. We take $A=1.1 \times 10^{-17} \mathrm{~cm}$ molecule ${ }^{-1}$, independent of ice composition (Gerakines et al. 1995), and thus find $N$ (solid $\mathrm{CO})=6.5 \times 10^{17} \mathrm{~cm}^{-2}$. The main source of uncertainty here is in $A$, which is about $10 \%$. CO in polar ices contributes $3.5 \times 10^{17} \mathrm{~cm}^{-2}$ to the total column, and the apolar

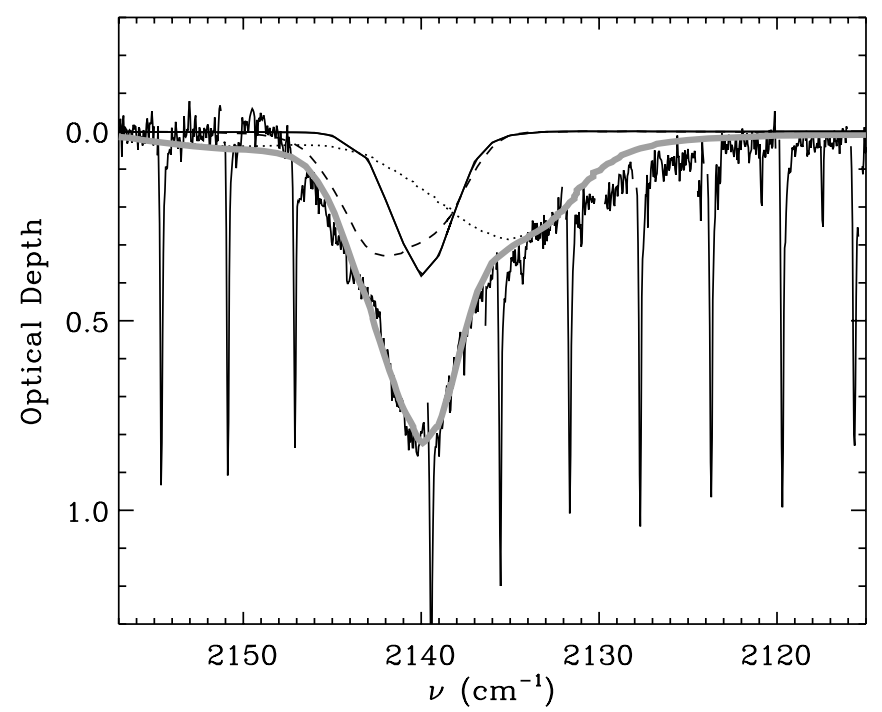

FIG. 5.-Observed CO absorption band of L1489 IRS, fitted by a combination of three laboratory ices. Dotted curve: Polar $\mathrm{H}_{2} \mathrm{O}: \mathrm{CO}=4: 1$ ( $T=50 \mathrm{~K}$ ) ice, to account for the long-wavelength wing. Dashed curve: Apolar $\mathrm{N}_{2}: \mathrm{O}_{2}: \mathrm{CO}_{2}: \mathrm{CO}=1: 5: 0.5: 1(T=10 \mathrm{~K})$ ice, for the short wavelength wing. Solid line: Pure CO ice ("CDE"'shape; $T=10 \mathrm{~K}$ ) that fits the central peak. The thick gray line is the sum of these components. 
components at 2140 and $2143 \mathrm{~cm}^{-1}$ each contribute $1.5 \times 10^{17} \mathrm{~cm}^{-2}$.

\section{DISCUSSION}

\subsection{An Infalling Disk}

The astrophysical meaning of the apparent two-component temperature structure seen in the ${ }^{13} \mathrm{CO}$ rotation diagram (Fig. 3) requires further investigation. For high-mass protostars it was found that similar rotation diagrams can be "mimicked" by power-law models of spherical envelopes (van der Tak et al. 2000).

For L1489 IRS, the detection of molecular gas at a range of temperatures and redshifted velocities could indicate the presence of infalling gas at a range of radii from the protostar. Indeed, a 2000 AU radius, contracting, disklike structure was found in millimeter-wave interferometer data (Hogerheijde \& Sandell 2000). In a detailed follow-up study, Hogerheijde (2001) adopts a flared-disk model based on Chiang \& Goldreich (1997), with a radial power-law distribution for the temperature,

$$
T=34(R / 1000 \mathrm{AU})^{-0.4} \mathrm{~K},
$$

and a density distribution that has a power-law drop-off with radius and a vertical exponential drop-off with scale height $h$,

$$
\rho(R, z)=\rho_{0}(R / 1000 \mathrm{AU})^{-1.5} \mathrm{e}^{-z^{2} / h^{2}} \mathrm{~kg} \mathrm{~cm}^{-3} .
$$

The scale height $h$ is assumed to be a simple function of $R$, $h=R / 2$. An inward-directed radial velocity field, described as

$$
V_{\text {in }}=1.3(R / 100 \mathrm{AU})^{-0.5} \mathrm{~km} \mathrm{~s}^{-1},
$$

is inferred, in addition to Keplerian rotation around a 0.65 $M_{\odot}$ central star.

Can this contracting-disk model, based on (sub-)millimeter emission observations with angular resolution of $4^{\prime \prime}-$ $8^{\prime \prime}$, reproduce the observed infrared $\mathrm{CO}$ absorption-line profiles measured along a pencil beam? The absorption lines are modeled with the radiative transfer code of Hogerheijde \& van der Tak (2000); the high densities in the disk ensure LTE excitation for the lines involved, and line trapping is neglected in the excitation calculation. The model spectra include dust opacity at a standard gas/dust ratio, as well as an $N(\mathrm{CO})=1 \times 10^{18} \mathrm{~cm}^{-2}$ column of cold foreground material (15 K; Hogerheijde 2001); neither factor affects the spectra in any significant way. The calculated spectrum is convolved with a Gaussian of FWHM $=12 \mathrm{~km} \mathrm{~s}^{-1}$, which is the NIRSPEC instrumental resolution.

While keeping all other parameters the same as in Hogerheijde (2001), we find that the assumed density profile sensitively influences the wings of the ${ }^{12} \mathrm{CO}$ lines. This is enhanced by the fact that we are observing the flared disk of L1489 IRS at an inclination between $60^{\circ}$ and $<90^{\circ}$ (see Padgett et al. 1999) and that the pencil beam crosses the disk at a few scale heights. Small changes in the density profile, induced, for example, by the thermal structure, have a large effect on the absorption-line profile. In the model of Hogerheijde (2001), the scale height increases linearly with distance from the star, and thus the density $\rho(l)$ along the line of sight $l$ follows the density in the midplane (eq. [2]), reduced by a factor $e^{-4 / \tan ^{2}(\alpha)}$, with $\alpha$ being the inclination. Here we include the effect of density variations, or deviations from the adopted scale height $h=R / 2$, by relaxing the values of the density along the line of sight $l$ by fitting $\rho(l)=\rho_{0}(1 / 1000 \mathrm{AU})^{-p}$ to the data.

This initial model successfully fits the peak velocity and depth of both high- and low- $J{ }^{13} \mathrm{CO}$ lines (Fig. 6). Its rotation diagram is quite different from that of the curve-ofgrowth analysis (Fig. 3), showing that rotation diagrams must be interpreted with great care. Our model also matches the range of velocities observed in the red wings of the ${ }^{12} \mathrm{CO}$ lines when taking $p=0.55 \pm 0.15$. This is a much shallower density profile than that derived from millimeter-wave data ( $p=1.5$; eq. [2]), and it indicates that the scale height increases more than linearly, i.e., the disk flares more than assumed in Hogerheijde (2001). With this result, it is possible to determine the important relation of disk scale height as a function of $R,\left[h(R)=a \cdot R^{b}\right]$ but only if the disk inclination is a priori known. Unfortunately, the inclination is not better constrained than within the range of $60^{\circ}$ and $<90^{\circ}$ imposed by near-infrared data (Padgett et al. 1999). We therefore cannot distinguish between low and high values of $a$ and the corresponding high and low inclinations. In either case, the total ${ }^{12} \mathrm{CO}$ column along the pencil beam is $1.2 \times 10^{19} \mathrm{~cm}^{-2}$, with $58 \%$ of the CO mass at a temperature of $T=20-60 \mathrm{~K}, 15 \%$ at $60-90 \mathrm{~K}$, and $27 \%$ at $>90 \mathrm{~K}$. This result is of importance in $\S 4.3$, in the interpretation of the solid $\mathrm{CO}$ observations, and in particular in assessing the thermal history of ices. The total column of our model is in good agreement with the column derived from the visual extinction $\left(1.4 \times 10^{19} \mathrm{~cm}^{-2} ; \S 3.1\right)$. It is also of the same order of magnitude as the total column through the midplane, calculated from dust and line emission $\left(N[C \mathrm{C}]=6 \times 10^{18} \mathrm{~cm}^{-2}\right.$; Hogerheijde 2001), and confirms the relatively edge-on orientation of the disk.

However, apart from these successes, the ${ }^{12} \mathrm{CO}$ lines show that our infalling-disk model produces too much warm gas at high velocities (Fig. 6). The ${ }^{12} \mathrm{CO}$ lines are a factor of 2.5 deeper, and, in contrast to ${ }^{13} \mathrm{CO}$, they peak at a too high a velocity $\left(10 \mathrm{~km} \mathrm{~s}^{-1}\right)$ with respect to the observations. In principle, one could make the ${ }^{12} \mathrm{CO}$ lines less deep by assuming that $\sim 1 \%$ of the original, unextincted continuum flux (corresponding to $30 \%$ of the extincted continuum) reaches the slit without passing through the disk, by scattering on large grains. The shift in peak velocity, however, requires a solution of a more fundamental origin. Perhaps the infall velocity function is shallower, and the disk is more rotationally supported, at lower radii. The amount of warm gas at high velocities can also be lowered by assuming that only part of the disk participates in the high-velocity inflow, such as a thin hot surface layer, or gas accelerated in magnetic field tubes directed from the inner disk to the stellar photosphere. Such a two-component model is consistent with the rotation diagram derived from the curve of growth (Fig. 3) and also with the low observed mass accretion rate. If we take the inflow at face value and assume that the entire disk participates, the mass accretion rate would be $10^{-6} M_{\odot}$, generating $7 L_{\odot}$ in accretion luminosity. The star's $L_{\text {bol }}$ is estimated at $3.7 L_{\odot}$, which also contains the stellar luminosity. It is therefore indeed likely that the mass accretion onto the star is significantly lower, as is also traced through the lack of the hydrogen $\operatorname{Pf} \beta$ emission line in our spectrum (2148.8 $\mathrm{cm}^{-1}$; Fig. 1) and the weakness of $\mathrm{Br} \gamma$ emission (Muzerolle, Hartmann, \& Calvet 1998). 

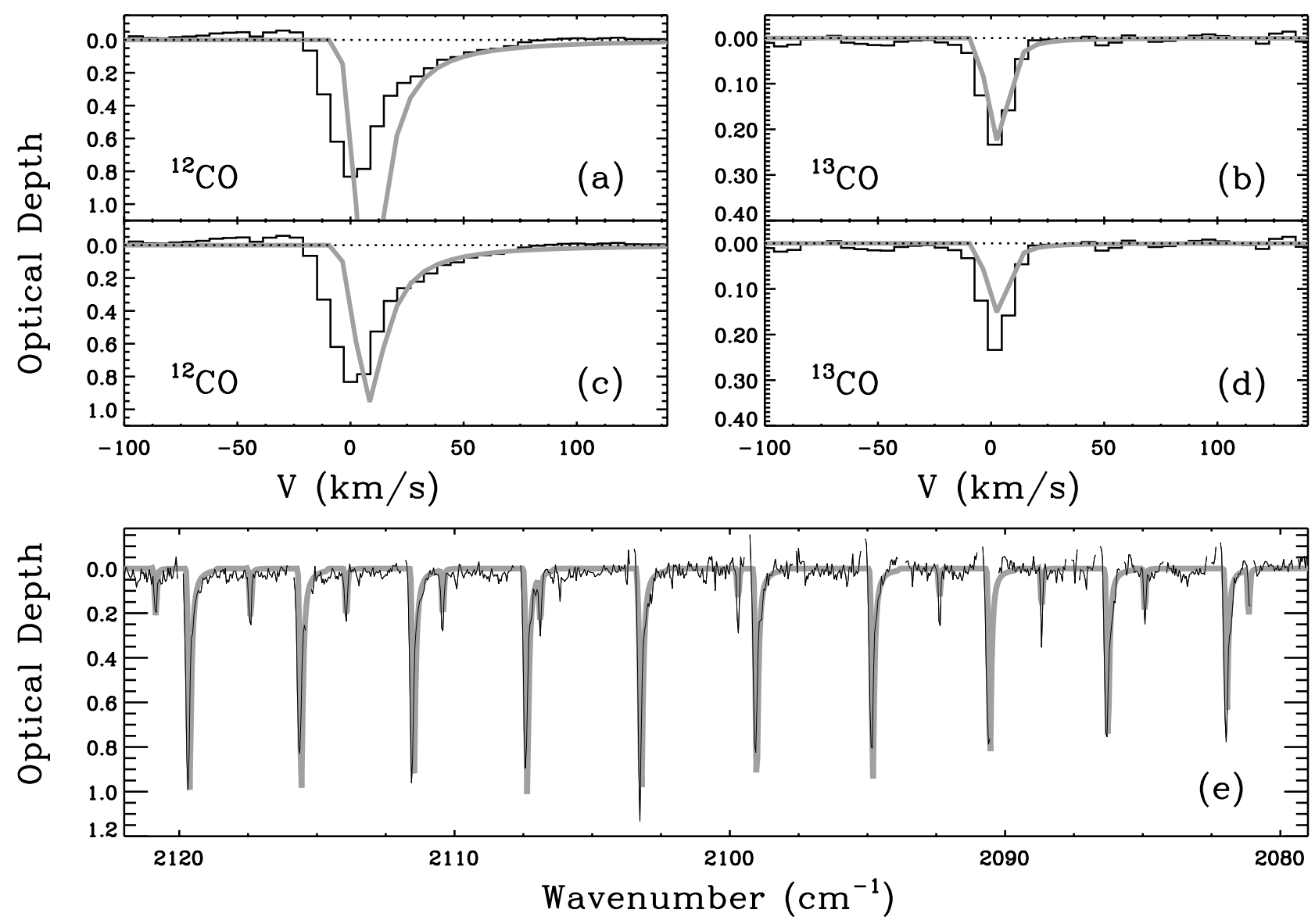

FIG. 6.-Comparison of the observed spectrum of L1489 IRS with an infalling-disk model. The histograms in panels $a$ and $b$ represent the average of the observed ${ }^{12} \mathrm{CO} P(6)-P(15)$ lines and all ${ }^{13} \mathrm{CO}$ lines, respectively, corrected for the source and earth velocity $\left(43 \mathrm{~km} \mathrm{~s} \mathrm{~s}^{-1}\right)$. The smooth thick line in these two panels is the collapsing-disk model, averaged over the same lines. In panels $c$ and $d$, the same averaged observed spectra and model are plotted, but now assuming that in the model $1 \%$ of the unextincted stellar continuum emission does not pass through the disk. Panel $e$ shows the latter model (thick gray line) compared with a portion of the nonaveraged L1489 IRS spectrum.

\subsection{Binarity?}

An entirely different explanation for the line profiles may lie in the possibility that L1489 IRS is a protobinary system. A protobinary nature of L1489 IRS is suggested by various pieces of evidence (Lucas, Blundell, \& Roche 2000; Wood et al. 2001 and references therein). The presence of a quadrupolar outflow system is inferred from $K$-band polarization images, $\mathrm{C}^{18} \mathrm{O}$ emission-line profiles, Herbig-Haro knots that are scattered throughout the L1489 IRS environment, and a very complex near-infrared scattered-light pattern. Threedimensional models, in which the axisymmetry of the infalling circumstellar envelope is broken by multiple outflow cavities that are perpendicular to each other, are able to account for the observed morphology.

The putative binary itself, however, has not been resolved so far. An upper limit on the projected separation has been set at $<20$ AU from near-infrared images (Padgett et al. 1999). If the CO absorption-line profile is in any way related to a binary system, then the large observed velocities $(\sim 23$ $\left.\mathrm{km} \mathrm{s}^{-1} ; \S 3.1\right)$ may indeed favor a close binary system. The line profile is then expected to vary on a timescale of a few months, which can easily be tested. In this case, much of the observed warm gas might be present in two small circumstellar disks, which are in a close orbit around each other. Some of the warm gas may also be present at low density in the central cavity created by the binary. The large column of cold gas may originate in the circumbinary disk. The binary extracts momentum from the 2000 AU circumbinary disk, setting up the inward motion seen in millimeter-wave emission lines. We leave further investigation of this topic for future studies.

\subsection{The Origin and Evolution of Ices}

In order to establish if the solid $\mathrm{CO}$ observed toward L1489 IRS originates in foreground clouds or in a circumstellar (or binary) disk, it is worthwhile to compare it with ices observed in lines of sight not affected by star formation. Observations of field stars obscured by intervening quiescent material of the Taurus molecular cloud have revealed that solid $\mathrm{CO}$ is not present when the extinction $A_{V} \lesssim 5$ (see, e.g., Teixeira \& Emerson 1999). The solid CO toward L1489 IRS therefore cannot be associated with foreground clouds, which have a gas column of $N\left({ }^{12} \mathrm{CO}\right)=1 \times 10^{18}$ $\mathrm{cm}^{-2}$ (Hogerheijde 2001), corresponding to $A_{V} \sim 2$.

Thus, the solid CO must be present in the disk of L1489 IRS. The absorption profile is intriguingly different from that seen in quiescent clouds. The broad red wing has a depth of $\sim 30 \%$ with respect to the narrow $2140 \mathrm{~cm}^{-1}$ peak, which is significantly more than that toward all measured background stars (10\%; Chiar et al. 1995). This may well be an effect of thermal processing along the L1489 IRS line of sight, because the sublimation temperatures of polar and 
apolar ices causing the broad and narrow features, respectively, are very different ( 90 vs. $18 \mathrm{~K}$ ). However, a chemical origin of an increased abundance of polar ices in disks cannot be excluded, because the apparently edge-on system Elias 18 in the Taurus molecular cloud has an extremely large CO depletion factor (solid/[gas + solid] 100\% vs. $7 \%$ for L1489 IRS), but a deep red "polar" CO wing is present as well (Shuping et al. 2001; Chiar et al. 1998). On the other hand, energetic processing may take place even in the cold disk of Elias 18 (Whittet et al. 2001). Clearly, it is necessary to observationally characterize the ices in circumstellar disks in much more detail.

If, for now, we assume the sublimation scenario, we can do some general extrapolations, which can be compared with the results of our gas-phase study (§ 4.1). By scaling the long-wavelength wing of solid $\mathrm{CO}$ of background field stars to that of L1489 IRS, we find that a column of $6 \times 10^{17}$ $\mathrm{cm}^{-2}$ of $\mathrm{CO}$ has evaporated from the apolar ice component, in the part of the L1489 IRS disk along the pencil absorption beam where $T<90 \mathrm{~K}$ (the sublimation temperature of polar ices). Thus, the column of solid $\mathrm{CO}$ that went from the quiescent cloud into building this part of the disk is $12.5 \times 10^{17} \mathrm{~cm}^{-2}$. Extrapolating this further, we use the observed CO depletion factor of 30\% toward field stars behind the Taurus molecular cloud (Chiar et al. 1995) to calculate that the original quiescent gas column must have been of the order of $3 \times 10^{18} \mathrm{~cm}^{-2}$. Adding the evaporated column, the expected present-day gas column at $T<90 \mathrm{~K}$ is $3.6 \times 10^{18} \mathrm{~cm}^{-2}$. This is of the same order of magnitude as the $\mathrm{CO}$ column below $90 \mathrm{~K}$ in our collapsing-disk model $\left(N[\mathrm{CO}]=8.7 \times 10^{18} \mathrm{~cm}^{-2}\right)$, which may indicate that no chemical change in the apolar-to-polar $\mathrm{CO}$-ice ratio and no significant additional depletion has occurred in the evolution from quiescent Taurus molecular cloud material to the formation of the L1489 IRS disk. This contrasts strongly with the very large depletions found in the (older) disks of $\mathrm{T}$ Tauri stars (Dutrey et al. 1997). The low CO depletion along the pencil beam toward L1489 IRS (7\%) and the supposed signs of thermal processing (see below) may be due to the fact that our line of sight does not cross the disk midplane, i.e., the system is not exactly edge-on. The ice processing we see takes place higher in the disk atmosphere, perhaps in the warm layer below the superheated dust layer responsible for millimeter-wave line emission (van Zadelhoff et al. 2001). It must be noted that in the model of Hogerheijde (2001), the gas temperatures are higher than $25 \mathrm{~K}$, prohibiting the formation of apolar ices and large $\mathrm{CO}$ depletions anywhere in the disk. The observed presence of apolar $\mathrm{CO}$ ices thus indicates that, as suggested in $\S 4.1$, the line of sight may cross the cold, rotationally supported disk interior not traced in the observations and infall model of Hogerheijde (2001).

Apart from evaporation of apolar ices, other hints of thermal processing include the aforementioned absence of the $2150 \mathrm{~cm}^{-1}$ absorption ( $\S 3.2$ ), which occurs in cold, unprocessed, polar $\mathrm{CO}$ ices but disappears at temperatures $T>50 \mathrm{~K}$. Also, the blue apolar wing may be a consequence of thermal processing. If the central $2140 \mathrm{~cm}^{-1}$ peak is due to a mixture of $\mathrm{O}_{2}, \mathrm{~N}_{2}$, and $\mathrm{CO}$, instead of pure $\mathrm{CO}$ (spectroscopically these cannot be distinguished), thermal or energetic processing (UV radiation from the interstellar radiation field, $\mathrm{UV}$ induced by $\mathrm{H}_{2}$ cosmic-ray collisions, or direct hits by cosmic rays) could efficiently produce $\mathrm{CO}_{2}$. This could cause the band to broaden and shift to the position of the observed blue wing. Chemical models indicate that energetic processing of molecules in disks takes place on a timescale of $10^{6} \mathrm{yr}$ (Aikawa et al. 1999), which is somewhat longer than the age of the disk of L1489 IRS $\left(\sim 5 \times 10^{5} \mathrm{yr}\right)$. This, however, applies to the disk midplane, and the timescale may well be shorter in the lower density, higher disk layers that our observations of L1489 IRS trace. A possible problem with the energetic processing interpretation is the absence of a feature adjacent to the short-wavelength side of the $\mathrm{CO}$-ice band, usually attributed to energetically produced $\mathrm{C} \equiv \mathrm{N}$ bondings (Whittet et al. 2001). Another spectroscopic tracer of thermal processing is the signature of crystallization in the band profiles of $\mathrm{H}_{2} \mathrm{O}$ and $\mathrm{CO}_{2}$ ices. Our infalling-disk model predicts that only $15 \%$ of the gas is within the temperature range at which ices crystallize $(60-90 \mathrm{~K})$, and thus crystallization is not expected to play a significant role in the disk of L1489 IRS. This model prediction can be tested with future high-quality $\mathrm{H}_{2} \mathrm{O}$ and $\mathrm{CO}_{2}$ spectra of L1489 IRS.

In summary, several pieces of evidence indicate that the $\mathrm{CO}$ ices in the disk of L1489 IRS have undergone thermal or energetic processing. The strongest arguments are the low depletion factor and the low ratio of apolar to polar ices with respect to the quiescent Taurus molecular cloud material. This may be explained by the fact that the disk of L1489 IRS is seen at an angle and that our pencil absorption beam traces the warm upper disk layers.

\section{SUMMARY AND FUTURE WORK}

We have shown that valuable and unique information is obtained from high-resolution spectroscopy of the $\mathrm{CO}$ fundamental at $4.7 \mu \mathrm{m}$ toward the low-mass Class I protostar L1489 IRS in the Taurus molecular cloud. At a resolution of $R=25,000\left(12 \mathrm{~km} \mathrm{~s}^{-1}\right)$, this object shows a multitude of deep rovibrational absorption lines of ${ }^{12} \mathrm{CO}$, as well as ${ }^{13} \mathrm{CO}$ and $\mathrm{C}^{18} \mathrm{O}$. The isotopes trace large columns of warm and cold gas in the circumstellar disk at or within $3 \mathrm{~km} \mathrm{~s}^{-1}$ of the systemic velocity, while the ${ }^{12} \mathrm{CO}$ line profiles show warm gas that is redshifted at a range of velocities of up to $100 \mathrm{~km}$ $\mathrm{s}^{-1}$. Both the line depth of the isotopes and the extent of the redshifted warm gas seen in ${ }^{12} \mathrm{CO}$ are well explained by an infalling-flared-disk model with power laws for the temperature, infall velocity, and density (a small column of blueshifted gas seen in the ${ }^{12} \mathrm{CO}$ line wing, however, remains unexplained). These observations show that the inward motions, inferred on scales of several hundred AU through millimeter-wave interferometry, continue to within $0.1 \mathrm{AU}$ of the star, where the velocity model of Hogerheijde (2001) predicts inward velocities exceeding several tens of $\mathrm{km} \mathrm{s}^{-1}$. A detailed comparison, however, shows that the amount of warm, high-velocity, infalling gas is overestimated by our power-law infall model. Much of the observed gas must therefore be rotationally supported, and only a thin disk surface layer is infalling, or gas is accelerated along magnetic fields in the inner parts of the disk. High spatial resolution millimeter-wave observations (with ALMA) are needed to test our model, e.g., to refine the determination of the velocity field in the inner disk parts and the dependence of the disk scale height on radius $h(R)$. Finally, high spatial resolution infrared interferometer observations would be able to see if L1489 IRS is a close binary system ( $<20 \mathrm{AU})$, which is essential to assess the importance of this aspect to the observed line profiles and disk evolution. 
In the same spectrum, a deep CO-ice band is seen toward L1489 IRS, which, we conclude, originates in the disk. The high signal-to-noise ratio and spectral resolution allowed us to separate it from circumstellar CO absorption lines and to do a detailed comparison with laboratory mixtures. It is, for the first time, found that, besides the well-known longwavelength wing of polar $\mathrm{H}_{2} \mathrm{O}$ : $\mathrm{CO}$ ices and the central narrow absorption peak at $2140 \mathrm{~cm}^{-1}$, an additional, separate component is present on the blue side of the ice band. Both the central peak and the blueshifted component are due to $\mathrm{CO}$ in apolar ices, one to pure $\mathrm{CO}$ and one to a mixture of $\mathrm{CO}_{2}$ and perhaps $\mathrm{O}_{2}$ and $\mathrm{N}_{2}$. The relatively large depth of the long-wavelength polar wing relative to the apolar components may suggest that the ices in the disk of L1489 IRS are thermally processed with respect to the quiescent Taurus molecular cloud material, which is supported by the low depletion factor $(\sim 7 \%)$ in this line of sight. This is likely a result of the fact that we see the disk at an angle and that our pencil absorption beam traces the upper disk layers.

The present work shows that high spectral resolution 4.7 $\mu \mathrm{m}$ observations are a great tool to better understand protostellar disks, which define the initial conditions of planet and comet formation in both the solid and gas phases. This is an exploratory study and must be followed up by observing a larger sample of protostellar disks at $4.7 \mu \mathrm{m}$, to investigate the influence of parameters such as foreground contribution, disk inclination, age, and binarity on gas and ice-band profiles.

The UCLA NIRSPEC instrument team is acknowledged for constructing an excellent instrument. We thank $M$. Brown (Caltech, USA) for providing a procedure to rectify two-dimensional spectra in IDL, T. Teixeira (University of Aarhus, Denmark) for generously providing her compilation of laboratory $\mathrm{CO}$ spectra to us, and the anonymous referee for careful reading of the manuscript, which helped to clarify the description of several aspects of the analysis. The research of A. C. A. B. at the Caltech Submillimeter Observatory is funded by the NSF through contract AST 9980846. The research of M. R. H. is supported by the Miller Institute for Basic Research in Science. NASA support to G. A. B. is gratefully acknowledged. The authors wish to extend special thanks to those of Hawaiian ancestry on whose sacred mountain we are privileged to be guests. Without their generous hospitality, none of the observations presented herein would have been possible.

\section{REFERENCES}

Aikawa, Y., Umebayashi, T., Nakano, T., \& Miyama, S. M. 1997, ApJ, 486, L51

1999, ApJ, 519, 705

Baratta, G. A., \& Palumbo, M. E. 1998, J. Opt. Soc. Am. A, 15, 3076

Bohren, C. F., \& Huffman, D. R. 1983, Absorption and Scattering of Light by Small Particles (New York: Wiley), chap. 5

Carr, J. S., Mathieu, R. D., \& Najita, J. R. 2001, ApJ, 551, 454

Chiang, E. I., \& Goldreich, P. 1997, ApJ, 490, 368

Chiar, J. E., Adamson, A. J., Kerr, T. H., \& Whittet, D. C. B. 1995, ApJ, 455,234

Chiar, J. E., Adamson, A. J., \& Whittet, D. C. B. 1996, ApJ, 472, 665

Chiar, J. E., Gerakines, P. A., Whittet, D. C. B., Pendleton, Y. J., Tielens,

A. G. G. M., Adamson, A. J., \& Boogert, A. C. A. 1998, ApJ, 498, 716

Dutrey, A., Guilloteau, S., \& Guélin, M. 1997, A\&A, 317, L55

Ehrenfreund, P., Boogert, A. C. A., Gerakines, P. A., Tielens, A. G. G. M., \& van Dishoeck, E. F. 1997, A\&A, 328, 649

Elsila, J., Allamandola, L. J., \& Sandford, S. A. 1997, ApJ, 479, 818

Gerakines, P. A., Schutte, W. A., Greenberg, J. M., \& van Dishoeck, E. F. 1995, A\&A, 296, 810

Goldsmith, P. F., Langer, W. D., \& Velusamy, T. 1999, ApJ, 519, L173

Gómez, M. Whitney, B. A., \& Kenyon, S. J. 1997, AJ, 114, 1138

Hogerheijde, M. R. 2001, ApJ, 553, 618

Hogerheijde, M. R., \& Sandell, G. 2000, ApJ, 534, 880

Hogerheijde, M. R., \& van der Tak, F. F. S. 2000, A\&A, 362, 697

Hogerheijde, M. R., van Dishoeck, E. F., Blake, G. A., \& van Langevelde, H. J. 1998, ApJ, 502, 315

Kenyon, S. J., Calvet, N., \& Hartmann, L. 1993, ApJ, 414, 676

Kenyon, S. J., \& Hartmann, L. 1987, ApJ, 323, 714

Lucas, P. W. Blundell, K. M. \& Roche, P. F. 2000, MNRAS, 318, 526

McLean, I. S., et al. 1998, Proc. SPIE, 3354, 566

Mitchell, G. F., Maillard, J.-P., Allen, M., Beer, R., \& Belcourt, K. 1990, ApJ, 363, 554

Muzerolle, J., Hartmann, L. \& Calvet, N. 1998, AJ, 116, 2965

Myers, P. C., Fuller, G. A., Mathieu, R. D, Beichman, C. A., Benson, P. J., Schild, R. E., \& Emerson, J. P. 1987, ApJ, 319, 340
Myers, P. C., Heyer, M., Snell, R. L., \& Goldsmith, P. F. 1988, ApJ, 324, 907

Najita, J., Bergin, E. A., \& Ullom, J. N. 2001, ApJ, 561, 880

Padgett, D. L., Brandner, W., Stapelfeldt, K. R., Strom, S. E., Terebey, S., \& Koerner, D. 1999, AJ, 117, 1490

Palumbo, M. E. \& Strazzulla, G. 1993, A\&A, 269, 568

Rothman, L. S., et al. 1992, J. Quant. Spectrosc. Radiat. Transfer, 48, 469

Sandford, S. A., Allamandola, L. J., Tielens, A. G. G. M., \& Valero, G. J. 1988, ApJ, 329, 498

Saito, M. Kawabe, R., Kitamura, Y. \& Sunada, K. 2001, ApJ, 547, 840

Sato, S., Nagata, T., Tanaka, M., \& Yamamoto, T. 1990, ApJ, 359, 192

Schmitt, B., Greenberg, J. M., \& Grim, R. J. A. 1989, ApJ, 340 , L33

Shuping, R. Y., Chiar, J. E., Snow, T. P., \& Kerr, T. 2001, ApJ, 547, L161

Teixeira, T. C., \& Emerson, J. P. 1999, A\&A, 351, 292

Teixeira, T. C., Emerson, J. P, \& Palumbo, M. E. 1998, A\&A, 330, 711

Thi, W.-F., et al. 2001, ApJ, 561, 1074

Tielens, A. G. G. M.. \& Hagen, W. 1982, A\&A, 114, 245

Tielens, A. G. G. M., Tokunaga, A. T., Geballe, T. R., \& Baas, F. 1991, ApJ, 381, 181

Trotta, F. 1996, Ph.D. thesis, Univ. Joseph Fourier (Grenoble)

van der Tak, F. F. S., van Dishoeck, E. F., Evans, N. J., II, \& Blake, G. A. 2000, ApJ, 537, 283

van Zadelhoff, G.-J., van Dishoeck, E. F., Thi, W.-F., \& Blake, G. A. 2001, A\&A, 377, 566

Whitney, B. A., Kenyon, S. J., \& Gómez, M. 1997, ApJ, 485, 703

Whittet, D. C. B., Pendleton, Y. J., Gibb, E. L., Boogert, A. C. A., Chiar, J. E., \& Nummelin, A. 2001, ApJ, 550, 793

Willacy, K., Klahr, H. H., Millar, T. J., \& Henning, T. 1998, A\&A, 338, 995

Willacy, K., \& Langer, W. D. 2000, ApJ, 544, 903

Wilson, T. L., \& Rood, R. T. 1994, ARA\&A, 32, 191

Wood, K., Smith, D., Whitney, B., Stassun, K., Kenyon, S. J., Wolff, M. J., \& Bjorkman, K. S. 2001, ApJ, 561, 299 